\title{
Optimal management of pulmonary ground-glass opacity nodules
}

\author{
Yeon Wook Kim ${ }^{1,2}$, Choon-Taek Lee ${ }^{1,2}$ \\ ${ }^{1}$ Division of Pulmonary and Critical Care Medicine, Department of Internal Medicine, Seoul National University College of Medicine, Seoul, \\ Republic of Korea; ${ }^{2}$ Division of Pulmonary and Critical Care Medicine, Department of Internal Medicine, Seoul National University Bundang \\ Hospital, Seongnam, Republic of Korea \\ Correspondence to: Choon-Taek Lee, MD, PhD. Division of Pulmonary and Critical Care Medicine, Department of Internal Medicine, Seoul National \\ University Bundang Hospital, 82, Gumi-ro 173, Bundang-gu, Seongnam-si, Gyeonggi-do 13620, Republic of Korea. Email: ctlee@snu.ac.kr. \\ Provenance: This is an invited article commissioned by the Academic Editor Chenyang Dai, MD, PhD (Department of Thoracic Surgery, Shanghai \\ Pulmonary Hospital, School of Medicine, Tongji University, Shanghai, China). \\ Comment on: Lee HW, Jin KN, Lee JK, et al. Long-Term Follow-Up of Ground-Glass Nodules After 5 Years of Stability. J Thorac Oncol \\ 2019;14:1370-7.
}

Submitted Sep 25, 2019. Accepted for publication Oct 21, 2019.

doi: $10.21037 /$ tlcr.2019.10.24

View this article at: http://dx.doi.org/10.21037/tlcr.2019.10.24

On the basis of the promising results of lung cancer screening in recent large trials, low-dose chest computed tomography (CT) has been widely introduced in clinical practice for the early detection of lung cancer $(1,2)$. The increased use of CT for screening has also led to an increased detection of lung nodules, with a significant proportion of ground-glass opacity nodules (GGNs). Although some GGNs are transient, persistent GGNs have a high likelihood of causing lung adenocarcinoma; also, their nature is different from typical lung cancer, thereby indicating a separate disease entity. Most patients detected with persistent GGNs are never-smokers, women, of Asian origin, and relatively young, of which are in contrast to the classic risk factors of lung cancer. Accordingly, the management of GGNs is important, although it is complicated owing to the indolent course of GGNs and the heterogeneity in growth rates, thereby requiring long-term follow-up and frequent CT screening. Herein, we discuss the appropriate management of GGNs, mainly focusing on the interval and duration of follow-up and the timing and modality of treatment.

\section{Follow-up of GGNs}

In real-world clinical settings, a significant proportion of GGNs that clinicians encounter are transient, as they disappear spontaneously (3). Among persistent cases, most GGNs remain stable without any change in size or features for years. However, according to long-term followup studies, a considerable percentage of GGNs tend to gradually grow over time. The appearance and growth of a solid component is also an important feature during the follow-up of GGNs in addition to a simple growth in size. We summarized recent studies that analyzed >50 GGNs with available information regarding the follow-up period, change in size or solid component, and diagnostic results (4-18) (Table 1). Kobayashi et al. reported that approximately $20 \%$ of pure GGNs and $40 \%$ of part-solid GGNs gradually grew or showed an increase in their solid components (19). They found that all GGNs with a significant increase in size grew within 3 years, and suggested that a minimum of 3 years of follow-up is reasonable (7). In 2016, Kakinuma et al. reported the data of a prospective multicenter study that evaluated 1,229 GGNs. They showed that the 5-year probabilities of nodule growth of $\geq 2 \mathrm{~mm}$ were $14 \%$ for pure GGNs, 24\% for heterogeneous GGNs (defined as a GGN with solid components only in the lung window but not in the mediastinal window setting), and $48 \%$ for partsolid GGNs. The 5-year probabilities of the appearance of a solid component were $6 \%$ and $22 \%$ for pure GGNs and heterogeneous GGNs, respectively (15). The data from that study showed that some GGNs start to grow even after 3 years of stabilization. In a study by our group in 2013 , we found that 2 of 90 GGNs (2.2\%) showed significant growth after 4 years (9). Moreover, another recent study from our group that evaluated 453 GGNs revealed that 


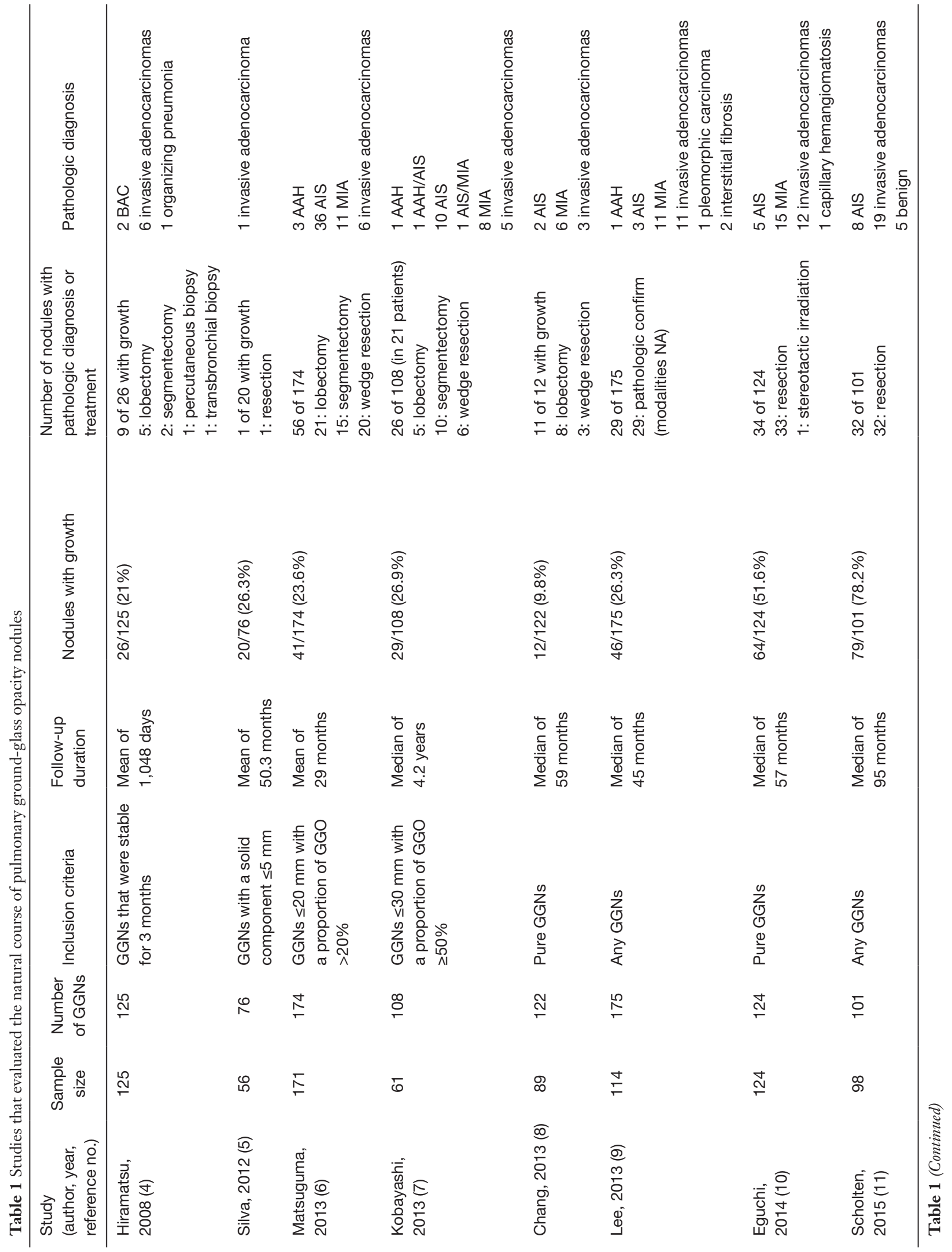




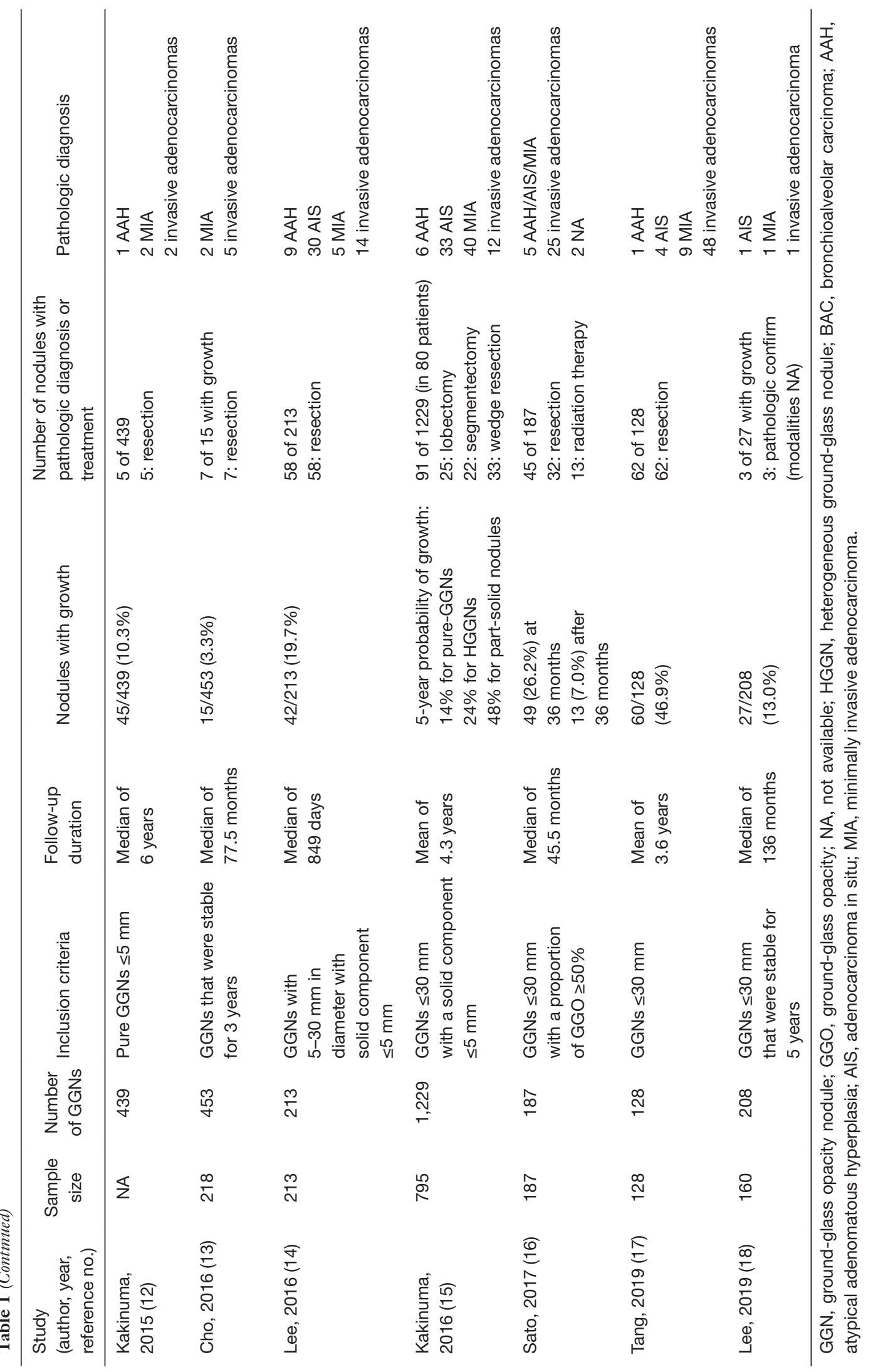


the frequency of subsequent growth of GGNs after 3 years of stabilization was $6.7 \%$ for person-based analyses and $3.3 \%$ for nodule-based analyses (13). Based on these recent data, the minimum suggested follow-up period for GGNs was extended to 5 years in the updated Fleischner Society guidelines in 2017 (20). While GGNs have a relatively high prevalence in Asian countries, studies in Caucasians revealed a much lower incidence of GGNs. For example, in the MILD trial from Italy, 76 GGNs were identified in 1,866 individuals who underwent baseline CT (5). In the NELSON trial in a Dutch-Belgian population, 264 GGNs were detected in 7,135 participants who underwent 4 rounds of low-dose CT (11).

Notably, in 2019, Lee et al. provided comparative information about the long-term natural course of GGNs after stabilization for 5 years (18). They evaluated 208 GGNs from 160 patients who had been stabilized for 5 years with a total of 10 years of follow-up. Of the evaluated GGNs, $27(13.0 \%)$ showed significant growth during a total follow-up of 136 months. In 8 of the 27 cases, there was a growth in size before the development of a new solid component. Moreover, in that study, approximately $70 \%$ of GGNs that showed growth after stabilization for 5 years had an initial size of $<6 \mathrm{~mm}$, and the growth was more prevalent in female patients with a smoking history of a few cigarette pack-years. As male sex, larger initial size, and smoking are previously reported risk factors for the growth of GGNs, Lee $e t a l$. as well as Kobayashi et al. (21) suggest that GGNs that grow after a long time of stabilization have clinical features different from those of GGNs in high-risk subjects with growth. The report from Lee et al. adds novel information to the field and indicates that a longer period of follow-up would be needed even in GGNs that were stabilized for a long time. Future research should focus on the identification of patients with GGNs who are at risk of showing GGN growth after long-term stabilization as well as optimizing the duration and interval of performing CT scans in such patients.

In 2015, Yankelevitz et al. reported the data of the I-ELCAP screening study that evaluated 57,496 participants who underwent repeated CT screenings; they revealed that GGNs of any size could be safely followed with CT at 12 -month intervals to assess the development of a solid component (22). Recently, Hammer et al. reported simulation results using the data from the NLST trial, suggesting that the follow-up interval for GGNs can be increased from 1 year to 3 years without a significant change in the clinical outcomes (23). Although it seems quite adequate to increase the interval for long-term CT screening for GGNs with no change, clinicians should always be aware of the possibility of growth in such nodules, and even small or stable GGNs should not be neglected. In addition, we have to take into account that the studies by Yankelevitz et al. and Hammer et al. enrolled mainly Caucasian participants and ever-smokers. Accordingly, different strategies for the optimal follow-up of GGNs might be needed in Asian countries where GGNs are more prevalent and are often observed in never-smokers.

\section{Treatment of GGNs}

The current recommendation for the treatment of GGNs is resection. However, the criteria for surgery vary among different guidelines. Results of recent studies indicate the importance of the presence and size of a solid component, of which are known to reflect the pathologically invasive component of adenocarcinoma (24). According to the recent Fleischner Society guidelines, resection is recommended for pure GGNs that grow or show the development of solid portions as well as for persistent part-solid nodules with solid portions of $\geq 6 \mathrm{~mm}$ (20). The guidelines of the American College of Chest Physicians recommend that GGNs that meet any of the following conditions should be resected: (I) GGNs with growth or development of new solid components, (II) pure GGNs $>10 \mathrm{~mm}$ with confirmed persistence, (III) part-solid GGNs $>8 \mathrm{~mm}$ with confirmed persistence, and (IV) part-solid GGNs $>15 \mathrm{~mm}$ without any follow-up (25). However, there is no generally accepted consensus regarding the optimal timing of surgery when GGNs show growth. Accordingly, it is still questionable whether urgent surgery is necessary for all such GGNs. Considering the commonly indolent course of GGNs even after the start of growth and the relatively low mortality of lung cancers presenting as GGNs compared to solid cancers (26), the life expectancy of patients with other medical conditions and the possibility of surgery-related complications should be considered.

The introduction of video-assisted thoracoscopic surgery has resulted in significant advances in the field of management of pulmonary nodules including GGNs. Currently, lobectomy is the modality of choice for resection of early lung cancer. A recent prospective study from the Japan Clinical Oncology Group revealed that the 5-year overall and relapse-free survival rates of patients who received lobectomy and lymph node dissection were $90.6 \%$ and $84.7 \%$, respectively (27). Recently, the use of limited 
resection such as segmentectomy or wide-wedge resection is increasing. Promising results have been reported regarding the performance of limited resection for relatively smaller GGNs that had outcomes similar to those of standard lobectomy (28). However, as limited resection is often associated with a higher recurrence rate for certain subtypes of early lung adenocarcinomas (29), lobectomy should be indicated for GGNs with a significant solid component; in addition, careful selection of patients who can undergo limited resection for GGNs is essential.

Stereotactic body radiation therapy (SBRT) and percutaneous ablation could be other options for local treatment of GGNs considered for resection. The study by Hammer et al. evaluated a simulation model for the treatment of GGNs with SBRT in patients aged $>77$ years, instead of lobectomy (23). The results revealed that, among patients who developed nodules that require treatment, the overall survival was higher for those treated with SBRT $(80 \%)$ than for those treated with surgery $(79 \%)$ and for those with no therapy (74\%). However, as the authors noted, we should take into account the fact that only a minority of GGNs had clinically significant malignancy in the study. Therefore, the mortality outcomes would have been driven not by recurrence but rather by treatmentrelated complication rates, which are higher for lobectomy than for SBRT. To date, there are limited data about the outcomes of SBRT or percutaneous ablation compared to lobectomy or limited resection for the treatment of early lung cancer. Unfortunately, 2 prospective studies that compared the outcomes of surgery and SBRT for early lung cancers were closed owing to slow accrual (30). Therefore, in future, the effects of treatment modalities need to be evaluated in real-world clinical settings rather than simulation models. To achieve this goal, large randomized prospective trials are needed. Another important limitation of SBRT and percutaneous ablation is that the pathologic results of the treated GGNs cannot be obtained, in contrast to resection, which can be used to perform the pathologic diagnosis and treatment simultaneously. This fact should be considered by corresponding clinicians because most patients with GGNs undergo resection without prior pathologic confirmation.

Regarding the proper management of GGNs, the multiplicity is also an important issue that should be taken into account. Approximately one-third of patients with GGNs have multiple nodules, which are usually similar in size and observed in different lobes. Generally, multiple GGNs are considered to be multiple synchronous lung cancer rather than a metastatic disease. A study by our group that investigated the genetic features of multiple GGNs resected from the same patients showed that a high frequency of discordant EGFR mutations (17 of 24, 70.8\%) could discriminate tumor clonalities (18 of $24,75 \%$ ) of multiple neoplastic GGNs (31). Accordingly, multiple GGNs should be treated as independent early lung cancers if they fulfill the criteria for resection, and such patients would have a high probability of undergoing multiple resection of the lungs. In these patients, initial resection of GGNs with limited resection can be an effective strategy to preserve the remnant pulmonary function. Non-surgical treatments such as SBRT or percutaneous ablation can be alternative options for patients with a high risk of complications or those who cannot undergo surgery.

In conclusion, despite the relatively long and indolent course, GGNs are generally heterogeneous, thereby making it difficult to predict the growth or development of a solid portion requiring treatment. In particular, a notable percentage of GGNs tend to grow even after a long time of stabilization. Therefore, understanding the distinct etiology-including the genetic features-along with more cumulative data on the long-term follow-up of such GGNs would allow the development of novel management strategies. In addition, future studies should focus on the selection of the GGNs for invasive treatment, while considering the timing and modality of therapy, especially in patients with multiple nodules. The relevant data regarding these issues would be essential for the optimal management of pulmonary GGNs.

\section{Acknowledgments}

None.

\section{Footnote}

Conflicts of Interest: The authors have no conflicts of interest to declare.

Ethical Statement: The authors are accountable for all aspects of the work in ensuring that questions related to the accuracy or integrity of any part of the work are appropriately investigated and resolved.

\section{References}

1. National Lung Screening Trial Research Team, Aberle 
DR, Adams AM, et al. Reduced lung-cancer mortality with low-dose computed tomographic screening. N Engl J Med 2011;365:395-409.

2. Horeweg N, Scholten ET, de Jong PA, et al. Detection of lung cancer through low-dose CT screening (NELSON): a prespecified analysis of screening test performance and interval cancers. Lancet Oncol 2014;15:1342-50.

3. Oh JY, Kwon SY, Yoon HI, et al. Clinical significance of a solitary ground-glass opacity (GGO) lesion of the lung detected by chest CT. Lung Cancer 2007;55:67-73.

4. Hiramatsu M, Inagaki $T$, Inagaki $T$, et al. Pulmonary ground-glass opacity (GGO) lesions-large size and a history of lung cancer are risk factors for growth. J Thorac Oncol 2008;3:1245-50.

5. Silva M, Sverzellati N, Manna C, et al. Long-term surveillance of ground-glass nodules: evidence from the MILD trial. J Thorac Oncol 2012;7:1541-6.

6. Matsuguma H, Mori K, Nakahara R, et al. Characteristics of subsolid pulmonary nodules showing growth during follow-up with CT scanning. Chest 2013;143:436-43.

7. Kobayashi Y, Fukui T, Ito S, et al. How long should small lung lesions of ground-glass opacity be followed? J Thorac Oncol 2013;8:309-14.

8. Chang B, Hwang JH, Choi YH, et al. Natural history of pure ground-glass opacity lung nodules detected by lowdose CT scan. Chest 2013;143:172-8.

9. Lee SW, Leem CS, Kim TJ, et al. The long-term course of ground-glass opacities detected on thin-section computed tomography. Respir Med 2013;107:904-10.

10. Eguchi T, Kondo R, Kawakami S, et al. Computed tomography attenuation predicts the growth of pure ground-glass nodules. Lung Cancer 2014;84:242-7.

11. Scholten ET, de Jong PA, de Hoop B, et al. Towards a close computed tomography monitoring approach for screen detected subsolid pulmonary nodules? Eur Respir J 2015;45:765-73.

12. Kakinuma R, Muramatsu Y, Kusumoto M, et al. Solitary Pure Ground-Glass Nodules $5 \mathrm{~mm}$ or Smaller: Frequency of Growth. Radiology 2015;276:873-82.

13. Cho J, Kim ES, Kim SJ, et al. Long-Term Follow-up of Small Pulmonary Ground-Glass Nodules Stable for 3 Years: Implications of the Proper Follow-up Period and Risk Factors for Subsequent Growth. J Thorac Oncol 2016;11:1453-9.

14. Lee JH, Park CM, Lee SM, et al. Persistent pulmonary subsolid nodules with solid portions of $5 \mathrm{~mm}$ or smaller: Their natural course and predictors of interval growth. Eur Radiol 2016;26:1529-37.
15. Kakinuma R, Noguchi M, Ashizawa K, et al. Natural History of Pulmonary Subsolid Nodules: A Prospective Multicenter Study. J Thorac Oncol 2016;11:1012-28.

16. Sato Y, Fujimoto D, Morimoto T, et al. Natural history and clinical characteristics of multiple pulmonary nodules with ground glass opacity. Respirology 2017;22:1615-21.

17. Tang EK, Chen CS, Wu CC, et al. Natural History of Persistent Pulmonary Subsolid Nodules: Long-Term Observation of Different Interval Growth. Heart Lung Circ 2019;28:1747-54.

18. Lee HW, Jin KN, Lee JK, et al. Long-Term Follow-Up of Ground-Glass Nodules After 5 Years of Stability. J Thorac Oncol 2019;14:1370-7.

19. Kobayashi Y, Mitsudomi T. Management of ground-glass opacities: should all pulmonary lesions with ground-glass opacity be surgically resected? Transl Lung Cancer Res 2013;2:354-63.

20. MacMahon H, Naidich DP, Goo JM, et al. Guidelines for Management of Incidental Pulmonary Nodules Detected on CT Images: From the Fleischner Society 2017. Radiology 2017;284:228-43.

21. Kobayashi Y, Ambrogio C, Mitsudomi T. Groundglass nodules of the lung in never-smokers and smokers: clinical and genetic insights. Transl Lung Cancer Res 2018;7:487-97.

22. Yankelevitz DF, Yip R, Smith JP, et al. CT Screening for Lung Cancer: Nonsolid Nodules in Baseline and Annual Repeat Rounds. Radiology 2015;277:555-64.

23. Hammer MM, Palazzo LL, Eckel AL, et al. A Decision Analysis of Follow-up and Treatment Algorithms for Nonsolid Pulmonary Nodules. Radiology 2019;290:506-13.

24. Lee KH, Goo JM, Park SJ, et al. Correlation between the size of the solid component on thin-section CT and the invasive component on pathology in small lung adenocarcinomas manifesting as ground-glass nodules. J Thorac Oncol 2014;9:74-82.

25. Gould MK, Donington J, Lynch WR, et al. Evaluation of individuals with pulmonary nodules: when is it lung cancer? Diagnosis and management of lung cancer, 3rd ed: American College of Chest Physicians evidence-based clinical practice guidelines. Chest 2013;143:e93S-e120S.

26. Hattori A, Matsunaga T, Hayashi T, et al. Prognostic Impact of the Findings on Thin-Section Computed Tomography in Patients with Subcentimeter Non-Small Cell Lung Cancer. J Thorac Oncol 2017;12:954-62.

27. Asamura H, Hishida T, Suzuki K, et al. Radiographically determined noninvasive adenocarcinoma of the lung: 
survival outcomes of Japan Clinical Oncology Group 0201. J Thorac Cardiovasc Surg 2013;146:24-30.

28. Cho JH, Choi YS, Kim J, et al. Long-term outcomes of wedge resection for pulmonary ground-glass opacity nodules. Ann Thorac Surg 2015;99:218-22.

29. Bains S, Eguchi T, Warth A, et al. Procedure-Specific Risk Prediction for Recurrence in Patients Undergoing Lobectomy or Sublobar Resection for Small ( $\leq 2 \mathrm{~cm}$ ) Lung Adenocarcinoma: An International Cohort Analysis. J Thorac Oncol 2019;14:72-86.

Cite this article as: Kim YW, Lee CT. Optimal management of pulmonary ground-glass opacity nodules. Transl Lung Cancer Res 2019;8(Suppl 4):S418-S424. doi:10.21037/ tlcr.2019.10.24
30. Chang JY, Senan S, Paul MA, et al. Stereotactic ablative radiotherapy versus lobectomy for operable stage I nonsmall-cell lung cancer: a pooled analysis of two randomised trials. Lancet Oncol 2015;16:630-7.

31. Chung JH, Choe G, Jheon S, et al. Epidermal growth factor receptor mutation and pathologicradiologic correlation between multiple lung nodules with ground-glass opacity differentiates multicentric origin from intrapulmonary spread. J Thorac Oncol 2009; 4:1490-5. 